\section{Grüne Gentechnik und nachhaltige Landwirtschaft}

\begin{abstract}
Akademien der Wissenschaften Schweiz (Akademien Schweiz) (Hg.): Gentechnisch veränderte Nutzpflanzen und ihre Bedeutung für eine nachhaltige Landwirtschaft in der Schweiz. Bern: Akademien der Wissenschaften der Schweiz 2013, 53 S., ISBN 978-3905870-33-6; kostenloser Download: http:// www.geneticresearch.ch/downloads/GrueneGentechnik BerichtC low.pdf
\end{abstract}

Bayerische Akademie der Wissenschaften (BAW) (Hg.): Pflanzenzucht und Gentechnik - In einer Welt mit Hungersnot und knappen Ressourcen. Rundgespräche der Kommission für Ökologie 40. München: Verlag Dr. Friedrich Pfeil 2012, 160 S., ISBN 978-389937-125-3, $€ 25,00$

\section{European Academies Science Advisory Council (EASAC): Planting the Future: Op- portunities and Challenges for Using Crop Genetic Improvement Technologies for Sus- tainable Agriculture. EASAC Policy Report 31, Halle/Saale: German National Academy of Sciences Leopoldina 2013, 59 S., ISBN 978-3-8047-3181-3; kostenloser Download: http://www.easac.eu/fileadmin/Reports/Plan- ting_the_Future/EASAC_Planting_the_Futu- re_FULL_REPORT.pdf}

B. Müller-Röber, M. Boysen, L. Marx-Stölting, A. Osterheide (Hg.): Grüne Gentechnik. Aktuelle wissenschaftliche, wirtschaftliche und gesellschaftliche Entwicklungen. Themenband der interdisziplinären Arbeitsgruppe Gentechnikbericht. Berlin-Brandenburgische Akademie der Wissenschaften (BBAW): Forschungsberichte Interdisziplinäre Arbeitsgruppen Band 31. Dornburg: Forum W - Wissenschaftlicher Verlag 2013, 282 S., ISBN 978-3-940647-05-4, € 39,90

\section{Sammelrezension von Martin Knapp und Rolf Meyer, ITAS}

In den letzten Monaten sind von Akademien der Wissenschaften vier Publikationen zur Grünen Gentechnik erschienen, und zwar in Deutschland, der Schweiz und auf europäischer Ebene. Die erklärte Zielsetzung ist sowohl die Politikberatung als auch die Information der breiten
Öffentlichkeit. Die aktuellen Herausforderungen Welternährung und umweltverträglichere Agrarproduktion bilden einen Ausgangspunkt der Argumentation in allen Berichten. Vor dem Hintergrund aktueller Herausforderungen wird die Grüne Gentechnik als ein wichtiger Lösungsansatz angeboten.

\section{Eine Kurzvorstellung}

Drei Berichte (Akademien Schweiz, BBAW, EASAC) sind in der klassischen Akademiearbeitsweise von Arbeitsgruppen (bzw. einer Projektgruppe) der jeweiligen Akademien erstellt worden und formulieren auf der Basis ihrer Problembeschreibung Handlungsempfehlungen.

Der Schweizer Akademiebericht ist in enger Zusammenarbeit mit dem Nationalen Forschungsprogramm NFP $59 \mathrm{zu}$ Nutzen und Risiken gentechnisch veränderter Pflanzen entstanden. Er behandelt mögliche Beiträge gentechnisch veränderter Pflanzen (GVP) zu einer nachhaltigen Landwirtschaft inklusive exemplarischer internationaler Erfahrungen (Beispiele aus USA, Kanada und Australien), die Herausforderungen beim Anbau von GVP sowie die spezielle Situation in der Schweiz (Moratorium für den kommerziellen Anbau von GVP durch Annahme einer Volksinitiative voraussichtlich bis Ende 2017). Er gelangt zu der Schlussfolgerung, dass gesetzliche Regulierungen in der Schweiz in Zukunft ein $\mathrm{Ne}-$ beneinander von Landwirtschaft mit und ohne GVP zulassen sollte, „ohne die Zusatzkosten übermäßig in die Höhe zu treiben und große administrative Hürden zu errichten" (Akademien Schweiz 2013, S. 49).

Der EASAC-Report analysiert internationale Erfahrungen mit GVP in Fallstudien (Anwendungen in Argentinien, Brasilien, Australien und Indien sowie Regulierung in Kanada) und Potenziale für Biotechnologie in Afrika (in $\mathrm{Zu}-$ sammenarbeit mit dem Network of African Science Academies - NASAC), evaluiert die Regulierungspolitik in der EU, um daraus Schlussfolgerungen und Handlungsempfehlungen abzuleiten. Im Zentrum steht die Forderung nach einer grundlegenden Änderung des EU-Regulierungs- 
ansatzes: „The aim should be to regulate the trait and/or product but not the technology in agriculture." (EASAC 2013, S. 2)

Der neue Gentechnikbericht der BBAW ist mittlerweile die dritte Ausgabe seit 2007. Seine Besonderheit ist, Problemfelder der Grünen Gentechnik in Deutschland mittels Indikatoren $\mathrm{zu}$ beschreiben, deren Daten fortgeschrieben werden und die Aussagen über den Stand und die Entwicklung der Grünen Gentechnik liefern sollen. Darüber hinaus liefert der Bericht einen ausführlichen, aktuellen Überblick über Stand von Wissenschaft und Technik (gentechnische und andere moderne biotechnologische Ansätze in der Pflanzenzüchtung), mögliche Auswirkungen auf Gesundheit und Umwelt, ökonomischen Nutzen, den politischen Rahmen in Deutschland und der EU sowie zu ethischen Bewertungen der Grünen Gentechnik. Im Mittelpunkt der Handlungsempfehlungen steht, dass „das wissenschaftliche und personelle Know-how auf dem Gebiet der Grünen Gentechnologie als Motor zukünftiger Innovationen langfristig in Deutschland gesichert werden" sollte sowie Risikobewertung wie ethische Bewertung ,keinesfalls pauschal bleiben, sondern sich auf den jeweiligen Einzelfall, den Anwendungskontext und das einschlägige Faktenwissen beziehen muss" (Müller-Röber et al. 2013, S. 24, 27). Schließlich werden in dem Band die Beiträge zu einer gemeinsamen Veranstaltung der interdisziplinären Arbeitsgruppe „Gentechnologiebericht" (IAG) der BBAW und der Vereinigung deutscher Wissenschaftler e.V. (VDW) aus dem Jahr 2010 zum Thema Welternährung dokumentiert. Die Beiträge stammen von Hans Rudolf Herren (Direktor des Millenniums Instituts und Co-Präsident des Weltagrarberichts), Bernd Müller-Röber (Sprecher der IAG der BBAW), Michael Krawinkel (Justus-Liebig-Universität Gießen und VDW) und Helmut Born (Mitglied des BioÖkonomieRats und Generalsekretär des Deutschen Bauernverbandes). Dies wird als ein Versuch bezeichnet, „dem Ziel des Herausarbeitens unterschiedlicher Überzeugungen einerseits und dem Bau eines Fundamentes für einen möglichen Brückenschlag andererseits ein wenig näher zu kommen“ (Müller-Röber et al. 2013, S.
157). Insgesamt zeichnet der Bericht sich dadurch aus, dass verschiedene Einschätzungen und offene Fragen fair dargestellt werden.

Der Band der BAW ist die Dokumentation eines sog. „Rundgesprächs“ mit 13 Vorträgen und 7 Diskussionsrunden, das im Juli 2011 in München stattfand. Es werden die drei Themenkomplexe „Ressourcen, Welternährung, Afrika“, „Pflanzenzüchtung: klassisch - gentechnisch“ und „Gesellschaft, Wirtschaft, Politik“ behandelt, ergänzt durch eine Zusammenfassung und Stellungnahme der Kommission für Ökologie der BAW. Entsprechend dem Themenspektrum haben die Vortragenden verschiedene disziplinäre Hintergründe, und mit Stefan Marcinowski (BASF) und Felix Prinz zu Löwenstein (Bund Ökologische Lebensmittelwirtschaft e.V.) sind auch zwei Repräsentanten von Interessengruppen vertreten. Die Beiträge halten sich eng an den Wortlaut der Vorträge. Zusammen mit den Diskussionsbeiträgen ergibt sich dadurch ein guter Einblick, wie um die Grüne Gentechnik auf verschiedenen Argumentationsebenen debattiert wird. Entgegen den unterschiedlichen Argumenten kommt die Kommission für Ökologie der BAW am Ende zu der Aussage, dass „Pflanzenzüchtung einschließlich der ,Grünen Gentechnik' ... das Hungerproblem der Welt nicht lösen [kann], sie ... jedoch wesentlich zur Lösung beitragen [wird]“ (BAW 2013, S. 156).

Im Folgenden wird auf ausgewählte gemeinsame Linien und unterschiedliche Einschätzungen der Berichte eingegangen.

\section{Moderne Pflanzenzüchtung und Grüne Gentechnik}

Vorherrschende Auffassung der in die moderne Biotechnologie involvierten Wissenschaftler ist, dass gentechnische Veränderungen nur ein weiterer Schritt in der langen Geschichte der Pflanzenzüchtung sind. So wird beim Vergleich von konventioneller Pflanzenzüchtung und Grüner Gentechnik nur ein kleiner Unterschied gesehen, denn in erster Linie würde die Vielfalt im Ausgangsmaterial erhöht bzw. eine neue nützliche Eigenschaft eingebracht (Akademien Schweiz 2013, S. 18). Die Vernei- 
nung neuer Risiken durch die Übertragung von artfremdem genetischem Material wird damit begründet, dass die praktische Erfahrung und wissenschaftlichen Untersuchungen bisher keine zusätzlichen Risiken für Mensch und Umwelt ergeben haben (Akademien Schweiz 2013, S. 19, 35; Müller-Röber et al. 2013, S. 15; EASAC 2013, S. 25). Die auf die bisherigen Erkenntnisse gestützte Argumentation dürfte aber nicht ausreichen, die öffentliche Wahrnehmung der Grünen Gentechnik grundsätzlich zu verändern, da die gesellschaftliche Diskussion gerade am grundsätzlichen Charakter der Technologie (z. B. Überschreitung von Artgrenzen, fehlende Rückholbarkeit) ansetzt und auf der Unterscheidung zwischen „künstlich“ und „natürlich" beruht (Darstellung der verschiedenen ethischen Bewertungen in Müller-Röber et al. 2013, S. 147-149).

Insbesondere die BBAW-Arbeitsgruppe beschreibt ausführlich neue Erkenntnisse und Methoden der Pflanzenbiotechnologie (Müller-Röber et al. 2013, S. 39ff.), die weit über den klassischen Gentransfer hinausgehen. In den anderen Berichten wird dies nicht so deutlich und umfangreich behandelt. Dieses breite Spektrum umfasst einerseits genotypische Selektionsverfahren, die beispielsweise in unterschiedlicher Weise mit Markern arbeiten. Zum anderen handelt sich um Ansätze wie cisgene Pflanzen, bei denen zwar die gleichen Methoden wie zur Erzeugung transgener Pflanzen verwendet werden, dabei aber nur arteigenes genetisches Material (oder das von kreuzbaren nahen Verwandten) benutzt wird, so dass cisgene Pflanzen grundsätzlich auch mit klassischer Züchtung (allerdings deutlich langwieriger) erzeugt werden könnten. Weiter gehören hierzu auch neue Ansätze wie „Gene Silencing“ und „Reverse Breeding“ (EASAC 2013, S. 7), wo das Endprodukt nicht mehr von einer konventionell gezüchteten Pflanze unterschieden werden kann. Es ist zu erwarten, dass diese Entwicklungen neue Regulierungsfragen aufwerfen werden (vgl. Bütschi et al. 2009).

Insgesamt wäre es wünschenswert, wenn eine deutlichere Abgrenzung zwischen Grüner Gentechnik (im Sinne gezielter Veränderungen des pflanzlichen Genoms durch Gentransfer) und moderner Pflanzenbiotechnologie vorgenommen würde. So bleibt ungeklärt, ob die Grüne Gentechnik mehr von den Entwicklungen in der modernen Pflanzenbiotechnologie profitiert oder umgekehrt, und wo tatsächlich die größeren Fortschritte zu erwarten sind.

Schließlich ist ein wiederkehrender Topos, dass keine technische Option ausgeschlossen werden sollte, teilweise mit dem Zusatz aus ideologischen Gründen (EASAC 2013, S. 1; BAW 2012, S. 156). Dies erscheint zunächst einleuchtend, verdeckt aber, dass bei begrenzten Forschungsmitteln nicht alle technischen Möglichkeiten der modernen Pflanzenzüchtung gleichermaßen verfolgt werden können. Damit bleibt die Frage zu beantworten, welche Optionen am vielversprechendsten sind. Entgegen dem technologiebezogenen Ansatz hat das Büro für Technikfolgen-Abschätzung beim Deutschen Bundestag (TAB) eine problem(lösungs)orientierte Herangehensweise bei der Suche nach zukunftsfähigen Agrartechnologien und Bewirtschaftungsweisen vorgeschlagen (Sauter 2008). Dies bedeutet, dass ausgehend von der jeweiligen landwirtschaftlichen Herausforderung das Problemlösungspotenzial und die Risiken verschiedener Ansätze (von veränderten Anbaumethoden über konventionelle Züchtung inklusive moderner markergestützter Selektionsmethoden bis zur Grünen Gentechnik) unvoreingenommen zu prüfen wären (ebd., S. 28).

\section{Grüne Gentechnik und nachhaltige Landwirtschaft}

Der Grünen Gentechnik wird durchweg attestiert, dass sie einen wichtigen Beitrag zu einer nachhaltigeren Landbewirtschaftung leisten kann. Wesentliche Begründung ist, dass mit gentechnischen Methoden entwickelte Kulturpflanzen einen umweltschonenderen und ertragreicheren Anbau ermöglichen, indem beispielsweise krankheitsresistente GVP den Einsatz von Pflanzenschutzmitteln reduzieren, der großflächige Anbau von schädlingsresistenten GVP Schädlingspopulationen nachhaltig verkleinern und der Anbau von herbizidtoleranten GVP 
eine verringerte Bodenbearbeitung ermöglichen (Akademien Schweiz 2013, S. 31).

Trotz dieser positiven Effekte bleibt die Frage, ob es sich hierbei nur um eine Symptombehandlung der Probleme der industriellen Landwirtschaft handelt (diskutiert im Beitrag Marx-Stölting in BAW 2012, S. 110). Der Gentechnikbericht der BBAW weist darauf hin, dass die Grüne Gentechnik in Europa zum Symbol negativer Aspekte der modernen, industrialisierten Landwirtschaft geworden ist und sie als ein weiterer Schritt in eine insgesamt als falsch empfundene Richtung gesehen wird (Müller-Röber 2013, S. 19).

Besonders ausgeprägt wird dagegen in der EASAC-Stellungnahme formuliert, dass negative Auswirkungen durch eine veränderte Anbaupraxis nicht den GVP zugerechnet werden sollten (EASAC 2013, S. 1) (was bei positiven Effekten nicht immer so gilt, s. u.). In zwei weiteren Stellungnahmen findet sich ebenfalls die Aussage, dass von GVP keine Umweltrisiken ausgingen, die nicht auch bei der konventionellen Landwirtschaft bestünden (Akademien Schweiz 2013, S. 5; BAW 2012, S. 156). Dagegen weist die BBAW richtigerweise darauf hin, dass die ökologische Bewertung von gentechnisch veränderten Sorten vom gewählten Vergleichsmaßstab, beispielsweise konventionelle Landwirtschaft oder Zielsetzungen des ökologischen Landbaus, abhängt (Müller-Röber et al. 2013, S. 106). Die Schwäche der Berichte mit explizitem Bezug zur Nachhaltigkeit ist deshalb, dass sie keine Bestimmung vornehmen, was unter nachhaltiger Landwirtschaft verstanden wird.

\section{4 Ökonomische Wirkungen der Grünen Gentechnik}

Ein weiteres wichtiges Argument sind die ökonomischen Vorteile, die Länder mit Nutzung von GVP erzielen. Zunächst ist richtig, dass sich Landwirte Vorteile vom GVP versprechen oder erfahren haben, sonst würden sie diese nicht anbauen. So wird beispielsweise zu Recht darauf hingewiesen, dass der Anbau von gentechnisch veränderter Baumwolle mit Insek- tenresistenz (Bacillus thuringensis (Bt) Baumwolle) in Indien den Gewinn von Kleinbauern auch über einen längeren Zeitraum (2002 bis 2008) deutlich gesteigert hat (EASAC 2013, S. 13; Müller-Röber et al. 2013, S. 120; Beitrag Qaim in BAW 2012, S. 89-96). Insgesamt ist die Situation aber nicht so einfach, wie eine Metaanalyse zu den ökonomischen Effekten des Bt-Baumwollanbaus zeigt, wonach aus den verschiedenen Einzelergebnissen ein genereller Trend nicht abgeleitet werden konnte, weil der tatsächliche bzw. mögliche Ertrag und Gewinn in vielfacher Weise beeinflusst wird, u. a. durch die vorhandene bzw. vorher verwendete Anbautechnik, die Schädlingsintensität, den stark schwankenden Saatgutpreis, die Konkurrenzsorten (Smale et al. 2006).

Ärgerlich ist deshalb, dass globale Abschätzungen von ökonomischen Vorteilen unkritisch übernommen werden. So wird von EASAC (2013, S. 12) ${ }^{1}$ ein kumulierter ökonomischer Gewinn von 72 Millionen US\$ durch den Anbau von GVP in Argentinien für den Zeitraum 1996 bis 2011 aus der Studie von Trigo (2011) zitiert. Hier wäre der Hinweis angebracht gewesen, dass diese Studie von ArgenBio veröffentlicht wurde, einer Organisation, die von Global Playern der Agrobiotechnologie finanziert wird. Ein Blick in die Studie hätte außerdem klar gemacht, dass 86 Prozent des Gesamtbetrages Bruttoeinnahmen sind, die aus der verstärkten Ausweitung der Sojaanbauflächen in Argentinien seit Einführung von gentechnisch verändertem Soja stammen. Der erhöhte Zuwachs wird aus dem Vergleich mit der durchschnittlichen Zuwachsrate im Zeitraum 1971 bis 1996 gewonnen (Trigo 2011, S. 15). Da in Brasilien die Ausdehnung des Sojaanbaus im Wesentlichen vor der Einführung von gentechnisch verändertem Soja erfolgte (EASAC 2013, S. 46), ist die Betrachtung der Sojaanbauflächenausweitung als Resultat der GVP-Einführung für Argentinien äußerst zweifelhaft. Hinzu kommt, dass es sich um Bruttoeinnahmen aus dem Sojaanbau handelt, also keine Angaben zum wirklich erzielten Gewinn gemacht werden.

Als ökonomischer Vorteil der Nutzung aller gentechnisch veränderter Sorten weltweit 
wird die Summe von 78,4 Mrd. US\$ für den Zeitraum 1996 bis 2010 aus der Studie von Brookes und Barfoot (2012) im BBAW-Bericht zitiert (Müller-Röber et al. 2013, S. 110). Dabei gibt es keinen Hinweis auf die Problematik dieser Zahl. Anhand einer älteren Version dieser Studie wurde gezeigt, dass die Basis oftmals Zahlen mit begrenzter Aussagekraft (z. B. einzelnes Jahr, kleine Region, kleine Stichprobe) sind, die trotzdem unabhängig von der Qualität der verfügbaren Daten für alle wichtigen transgenen Sorten und alle wichtigen Anbauländer zu volkswirtschaftlichen Werten hochgerechnet werden. Die resultierenden Zahlen sollten daher in einer seriösen Auseinandersetzung über die Potenziale der Grünen Gentechnik nur mit äußerster Vorsicht bzw. unter Kenntlichmachung ihrer jeweiligen Datenbasis, Herkunft und Qualität verwendet werden (Sauter 2008, S. 231-233).

\section{5 Ökologischer Landbau und Grüne Gentechnik}

In mehreren Beiträgen des BAW-Rundgesprächs wird betont, dass eine enge Kooperation zwischen ökologischem Landbau und moderner Pflanzenzüchtung einschließlich Gentechnik für die zukünftige Entwicklung wichtig sein könnte (BAW 2012, S. 156). Diese Vorstellung einer „Versöhnung“ im Rahmen einer nachhaltigen Landbewirtschaftung stellt allerdings unzureichend in Rechnung, dass die Technologien der Grünen Gentechnik sich nicht gut mit dem Prinzip des ökologischen Landbaus und seinen Vorstellungen von der zu wahrenden Integrität der Pflanzen vertragen, abgesehen von den EU-Regeln für ökologische Lebensmittel, die eine Nutzung Grüner Gentechnik ja explizit untersagen. Daneben stellt sich die grundsätzliche Frage, ob sich agrarökologische Ansätze einer nachhaltigen Intensivierung und umweltverträglichere Anbauelemente durch GVP gegenseitig ergänzen oder, als Ansätze einer komplexeren Gestaltung von Anbausystemen einerseits und tendenzieller Vereinfachung andererseits, eher in unterschiedliche Richtungen weisen.

\section{Patente und "offene Innovation“}

Teilweise wird thematisiert, dass Sortenzüchtung und Saatguthandel von gentechnisch veränderten Sorten zunehmend von wenigen, global agierenden Unternehmen dominiert werden, mit dem Resultat einer zunehmenden Abhängigkeit der Landwirte (Akademien Schweiz 2013, S. 41; Beitrag Marx-Stölting in BAW 2012, S. 107f.). Dies wird im Kontext der Globalisierung aller Wirtschaftsbereiche sowie als eine Folge der Kostensteigerungen für Forschung, Entwicklung und Zulassung gesehen (Akademien Schweiz 2013, S. 41). Hinzu kommt, dass das System der Züchterrechte zunehmend durch die Patentierung von Pflanzeneigenschaften unter Druck gerät (EASAC 2013, S. 31). Beispielsweise waren beim „Golden Rice“ Projekt (gentechnisch veränderter Reis mit Provitamin A für arme Bevölkerung in Entwicklungsländern) für 72 Patente freie Lizenzen zu organisieren (Potrykus in BAW 2012, S. 99).

Als Gegenmaßnahme wird eine stärkere Förderung der Agrarforschung und -züchtung an öffentlichen Forschungsinstitutionen vorgeschlagen (Akademien Schweiz 2013, S. 41; EASAC 2013, S. 2). Zusätzlich werden von EASAC neue Möglichkeiten öffentlich-privater Partnerschaften und der „offenen Innovation“ betont (EASAC 2013, S. 31). Wie diese „offene Innovation" ohne Patentrechte gestaltet werden soll, bleibt aber unklar.

\section{EU-Regulierung der Grünen Gentechnik}

Exemplarisch ist die Kritik, dass die europäische Gentechnik-Regulierung eine ideologische und keine wissenschaftliche Basis habe, und diese Regulierung den öffentlichen Sektor daran hindere, GVP für das Allgemeinwohl zu entwickeln, sowie kleine und mittlere Unternehmen benachteilige (Beitrag Potrykus in BAW 2012, S. 87). Entsprechend wird von EASAC gefordert, dass zukünftig die GVP nicht anhand der Gentechnik, sondern anhand der Eigenschaft bzw. des Produkts reguliert werden sollten, und dabei auf die Regulie- 
rungserfahrungen außerhalb Europas zurückgegriffen werden sollte (EASAC 2013, S. 2, 38). Nicht ausgesprochen wird, dass dies ein Zurück zur EU-Regulierung vor 2001 und den Verzicht auf Vorsorgeprinzip, Wahlfreiheit und Kennzeichnungspflicht bedeuten würde.

Dies wirkt etwas aus der Zeit gefallen, wo BASF und Monsanto gerade angekündigt haben, keine gentechnisch veränderten Sorten mehr für den europäischen Markt zu entwickeln. Dem liegt die Einschätzung zugrunde, dass sich an der EU-Regulierung sowie den verbreiteten öffentlichen Vorbehalten und dem organisiertem gesellschaftlichen Druck in absehbarer Zeit nichts grundlegend ändern wird. Dementsprechend erwartet der Bericht der BBAW, dass in Deutschland in den nächsten Jahren GVP keinen nennenswerten Anteil am Anbau einnehmen werden (Müller-Röber et al. 2013, S. 26).

\section{Fazit}

Zwischen den Berichten, die von in der modernen Biotechnologie bzw. Grünen Gentechnik engagierten Wissenschaftlern erstellt wurden (Akademien Schweiz, EASAC), und den von interdisziplinären Arbeitsgruppen erarbeiteten Publikationen (BAW, BBAW) sind deutliche Unterschiede zu erkennen. Aus ersteren ist zu erfahren, wie diese die Potenziale der Grünen Gentechnik einschätzen und welche Forderungen sie an die Regulierung haben. Die zweite Gruppe bemüht sich, eine faire Darstellung der wissenschaftlichen und gesellschaftlichen Kontroversen um die Grüne Gentechnik zu liefern. Dies schließt eine Diskussion der rechtlichen und politischen Rahmenbedingungen in Deutschland und der EU ein, die nicht grundsätzlich in Frage gestellt werden.

\section{Anmerkung}

1) In einem Annex (EASAC 2013, S. 51) werden zwar die methodischen Schwierigkeiten bei der Bestimmung sozioökonomischer Wirkungen kurz diskutiert, auf die Darstellung im Bericht hat dies aber keinen Einfluss.

\section{Literatur}

Bütschi, D.; Gram, S.; Haugen, J.M. et al., 2009: Genetically Modified Plants and Foods - Challenges and Future Issues in Europe. Final Report of the Joint EPTA Project. Berlin

Sauter, A., 2008: Transgenes Saatgut in Entwicklungsländern - Erfahrungen, Herausforderungen, Perspektiven. TAB-Arbeitsbericht Nr. 128, Berlin

Smale, M.; Zambrano, P.; Cartel, M., 2006: Bales and Balance: A Review of the Methods Used to Assess the Economic Impact of Bt Cotton on Farmers in Developing Economies. In: AgBioForum 9/3 (2006), S. 195-212

Trigo, E.J., 2011: Quince Anos de Cultivos Genéticamente Modificados en la Agricultura Argentina (Fifteen Years of Genetically Modified Crops in Argentine Agriculture). Argenbio; http://www.argenbio.org/ adc/uploads/15_anos_Estudio_de_cultivos_GM_en_ Argentina.pdf (download 11.7.13)

\section{$\ll 》$}

\section{TATuP mit neuer Homepage}

Die Zeitschrift „Technikfolgenabschätzung - Theorie und Praxis“ (TATuP) hat einen neuen Internetauftritt. Ab sofort finden Sie die Zeitschrift auf der Homepage http://www.tatup-journal.de.

Unter dieser Adresse steht Ihnen das Vollarchiv der Zeitschrift, gegliedert nach Ausgaben oder Schwerpunktthemen, kostenfrei zur Verfügung. 